(C) 2015 IEEE. Personal use of this material is permitted. Permission from IEEE must be obtained for all other uses, in any current or future media, including reprinting/republishing this material for advertising or promotional purposes, creating new collective works, for resale or redistribution to servers or lists, or reuse of any copyrighted component of this work in other works. 


\section{Estimation of the Flexibility Range in the Transmission-Distribution Boundary}

\author{
M. Heleno, R. Soares, J. Sumaili, R.J. Bessa, L. Seca \\ INESC TEC \\ Center for Power and Energy Systems \\ Porto, Portugal \\ mdheleno@inescporto.pt, rasoares@inescporto.pt, \\ jean.sumaili@inescporto.pt, ricardo.j.bessa@inesctec.pt, \\ lseca@inescporto.pt
}

\begin{abstract}
The smart grid concept increases the observability and controllability of the distribution system, which creates conditions for bi-directional control of Distributed Energy Resources (DER). The high penetration of Renewable Energy Resources (RES) in the distribution grid may create technical problems (e.g., voltage problems, branch congestion) in both transmission and distribution systems. The flexibility from DER can be explored to minimize RES curtailment and increase its hosting capacity. This paper explores the use of the Monte Carlo Simulation to estimate the flexibility range of active and reactive power at the boundary nodes between transmission and distribution systems, considering the available flexibility at the distribution grid level (e.g., demand response, on-load tap changer transformers). The obtained results suggest the formulation of an optimization problem in order to overcome the limitations of the Monte Carlo Simulation, increasing the capability to find extreme points of the flexibility map and reducing the computational effort.
\end{abstract}

Index Terms - Flexibility; power flow; smart grid; TSO-DSO cooperation; Monte Carlo simulation.

\section{INTRODUCTION}

Smart grids provide additional capabilities for the observability and controllability of the distribution grid (e.g., distributed storage, distributed generation, controllable loads) [1]. The Distribution System Operators (DSO) are investing in a new Information and Communications Technology (ICT) infrastructure that provides bi-directional control over small/medium-scale distributed generation and Distributed Energy Resources (DER) connected to the MV and LV grids [2].

In this paradigm, new market players, such as flexibility operators or aggregators, are emerging in several countries. Furthermore, the classical tap-changing control of the DSO is also another type of flexibility that can be requested by the Transmission System Operator (TSO) to solve voltage problems close to the transmission/distribution boundary (i.e., primary substation nodes).

The research leading to this work is being carried out as a part of the evolvDSO project (Development of methodologies and tools for new and evolving DSO roles for efficient DRES integration in distribution networks) www.evolvdso.eu. This project is funded by the European Commission under the seventh framework program (FP7) under grant agreement number 608732.

\author{
Manuel A. Matos \\ INESC TEC and Faculty of Engineering of the University of \\ Porto \\ Porto, Portugal \\ mam@fe.up.pt
}

The existence of DER in the distribution network operated by the DSO creates several challenges, such as: (a) the activation of these DER resources, by the TSO, might create technical problems in the distribution grid, thus some flexibility cannot be fully explored; (b) a close coordination between TSO and DSO is required in order to share this flexibility for different grid management tasks, such as voltage control, balancing, congestion management; (c) these new resources should be included into the planning task of both TSO and DSO (topic not addressed in this paper).

In distribution systems with high penetration of Renewable Energy Resources (RES), mainly wind and solar power, the occurrence of reverse power flows and voltage limits violations in the transmission grid might become frequent. In order to solve these local problems, the TSO can request control actions at the level of distribution grid, using the available levers (e.g., reactive power control, control-set points for DER). For instance, Pudjianto et al. [3] describe a framework named technical virtual power plant where the DSO seeks to maintain a fixed active and reactive power profile at the primary substation nodes; Schwerdfeger and Westermann [4] describe an algorithm that uses distributed storage as a resource to schedule DSO system operation, i.e. TSO and DSO negotiate a power flow schedule for each point of common coupling.

The TSO/DSO interaction is covered by the new ENTSOE Network Code "Operational Planning and Scheduling" [5], where it is stated that the TSO should monitor the ratios of active and reactive power at the interface between transmission systems and distribution networks. The TSO and DSO cooperation was also identified as a potential future role/services of the DSO in the EU project evolvDSO [6,7] and it is being studied at the European Commission level [8].

In this context, this paper proposes a new algorithm which estimates the flexibility range in each primary substation node for the next hours (time horizon defined by the available forecasted data, see [9] for instance). The main goal of this algorithm is to inform the TSO about the technically feasible 
flexibility of each distribution grid, in the boundary between transmission and distribution systems. This algorithm includes different levers (or flexibilities), such as demand response aggregators, flexible distributed generation and reactive power flow control from the DSO assets. With this information, the TSO knows in which primary substation nodes can request active and reactive power control to the DSO in order to solve technical problems in the transmission grid. It also enables an evaluation of the DER aggregators' impact on the transmission grid. The cost of activating the flexibility resources in the distribution grid is also included in the algorithm so that both TSO and DSO can perform a cost/benefit evaluation of the available levers.

The innovative algorithm proposed in this paper addresses a forthcoming scenario characterized by an increasing investment in ICT equipment and the emergence of new business models, such as demand response and flexibility aggregators.

This paper is organized as follows: section II presents the general framework for the flexibility range estimation; section III describes the Monte Carlo Simulation methodology; section IV presents numerical results for a radial distribution network with 211 buses; the paper ends with conclusions and topics for future development.

\section{FLEXIBILITY RANGE ESTIMATION FRAMEWORK}

The main goal, as depicted in Fig. 1, is to estimate the range of technically feasible active and reactive power flexibility (minimum and maximum values) at the primary substation nodes. This provides to the TSO information about how much flexibility can the distribution network "offer" for a time horizon defined for each hour. This information is essential to support the definition of control actions necessary to solve technical problems in the transmission network operation, such as those provoked by reverse power flows.

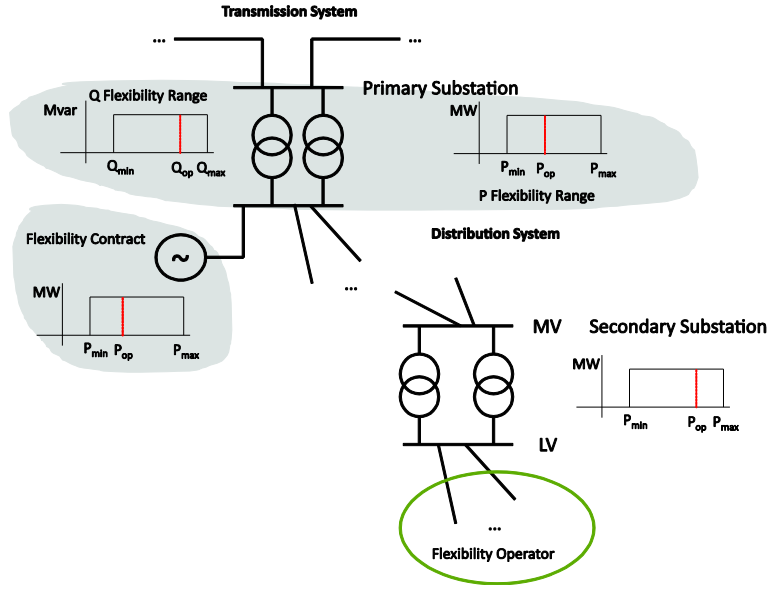

Figure 1. Flexibility range estimation in a distribution grid.

The flexibility in the TSO/DSO boundary nodes is estimated based on different types of flexibilities, such as:

- mid-term bilateral flexibility contracts: annual flexibility tenders between TSO and flexibility operators and other flexible resources (see in [10] the example of France);
- non-firm connection contracts: generators and large consumers that are willing to accept curtailment during some hours per year in order to get a connection license to the distribution network;

- flexibility bids at the level of MV/LV nodes, e.g., from local demand response aggregators [10];

- controllable resources connected to the distribution network and owned by the DSO, e.g., capacity banks, on-load tap changers (OLTC).

The methodology to obtain flexibility range estimation consists in three main steps:

1. Receive information regarding distribution flexibility offers, status of the resources owned by the DSO, flexibility bilateral contracts, and non-firm connection contracts at the secondary substation and MV levels. As illustrated by Fig. 1, this information will be used to construct an interval of flexibility (minimum and maximum $\mathrm{P}$ and $\mathrm{Q}$ power values) in each node of the MV network. Receive forecasted information about net-load for the distribution grid;

2. Execute the flexibility estimation algorithm proposed in section III.

3. Send information about the range of flexibility to a TSO-DSO common data sharing platform. This output can be used during the TSO-DSO negotiations for setting the minimum and maximum active/reactive power flow values in primary substations $[3,4]$ or by the TSO to activate flexibility available in the distribution grid.

A detailed description of these steps can be found in the system use case "Estimate flexibility range of the primary substations" elaborated in the framework of the EU project evolvDSO [11], using the IEC/PAS 62559 methodology [12].

\section{FLEXIBILITY ESTIMATION METHODOLOGY}

The proposed methodology aims at identifying the flexibility range in the boundary nodes between transmission and distribution systems for a period required by the TSO (typically with hourly intervals). The active and reactive power flexibility in these boundary nodes are determined considering the aggregated flexibility range available in $\mathrm{MV} / \mathrm{LV}$ nodes as well as in DSO controllable resources (e.g., capacity banks, on-load tap changer transformers).

The algorithm consists in performing a simulation comprising a significant number of power flow scenarios in the MV network based on random input variables associated with the flexibility resources. Each power flow scenario corresponds to an operating point in the primary substation. If the network limits are not violated, this operating point is feasible. All the feasible operation points form a P-Q admissible flexibility region in the TSO/DSO boundary node, as illustrated in Fig. 2. It is important to stress that the dependence between active and reactive power influences the P-Q flexibility region. 


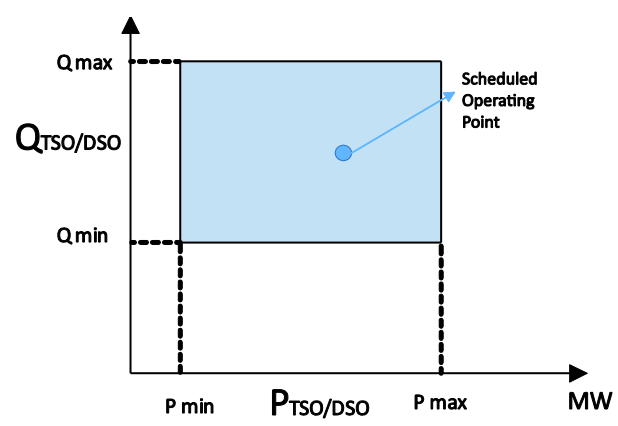

Figure 2. Flexibility range in a primary susbtation.

Each operating point encompasses a cost, which depends on the quantity of flexible resources that were activated in the MV network. Therefore, the flexibility region can be described in terms of a cost map, where different colors may represent different price intervals. Undoubtedly, this information allows TSO to deal with the problems in the transmission grid and making cost-effective decisions.

The algorithm, represented by a flowchart in Fig. 3, starts with a power flow calculation in order to determine the scheduled operating point on the flexibility map.

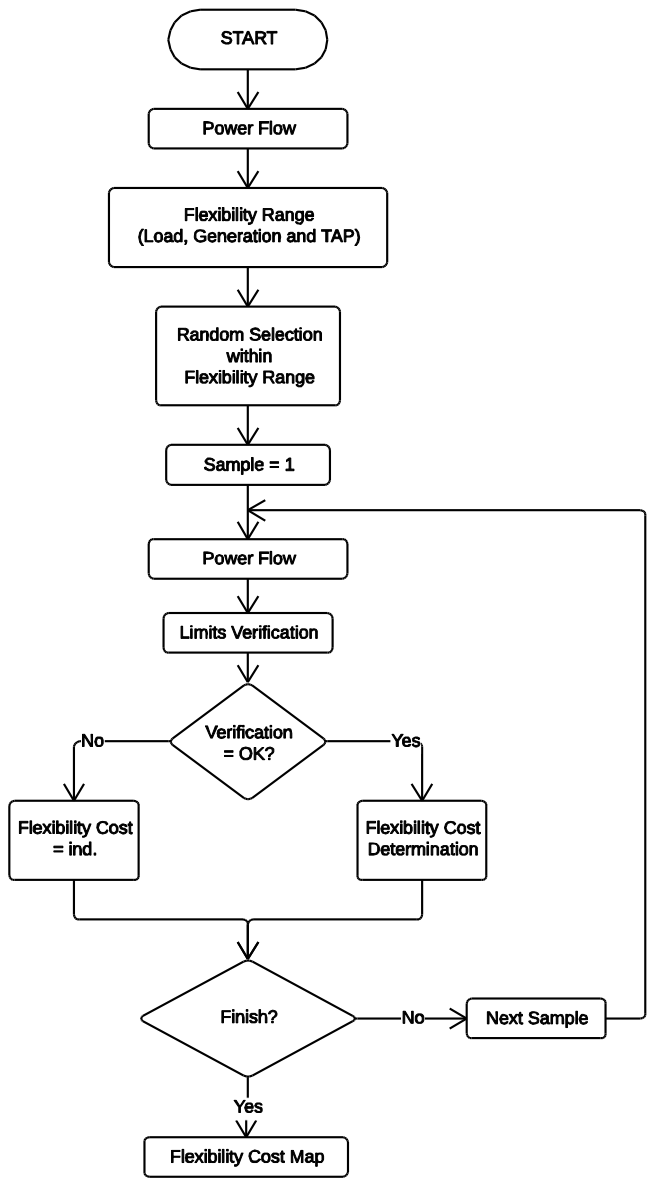

Figure 3. Flowchart of the flexibility estimation algorithm.

Then a predefined number of samples are extracted randomly. For each sample, a power flow calculation is performed to verify if it is feasible, i.e. there is no violated technical constraint. If the sample represents a feasible point, then the power exchange (active and reactive power) with the TSO network is plotted on the flexibility map with the identification of its activation cost. The procedure is repeated until all the samples have been analyzed. The random selection of the flexibility resources, the limits variation process as well as the price calculations are detailed in the following sub-sections.

\section{A. Flexibility range: Load, Generation and Tap}

For each power flow, the consumption/generation in the $\mathrm{MV} / \mathrm{LV}$ nodes are randomly selected within the maximum and minimum limits of the available flexibility resources. In this paper two approaches to perform a random selection were explored. In the first approach, an independent random variable was associated to each flexible resource (generation and load). On the other hand, in the second approach, a negative correlation of one between the generation and load in the same bus was considered, i.e., when the generation increases the load decreases.

Regarding the MV network controllable resources, it is assumed that DSO can change remotely the OLTC tap position in order to increase the flexibility range in the primary substation. Therefore, the discrete flexibility related to possible tap positions was included in the algorithm. For each OLTC, the tap position was randomly selected according to a discrete uniform distribution.

In the first method, in each power flow iteration $(k)$ the active and reactive power injected $\left(P_{n, k^{\text {flex }}}, Q_{n, k^{\text {flex }}}\right)$ in the flexible bus $n$ of the MV network were obtained through equation (1)-(2):

$$
\begin{aligned}
P_{n, k}^{f l e x}= & \left(P G_{n, k}^{\max }-P G_{n, k}^{\min }\right) \cdot \varphi_{n, k}^{P G}+P G_{n, k}^{\min } \\
& -\left(P L_{n, k}^{\max }-P L_{n, k}^{\min }\right) \cdot \varphi_{n, k}^{P L}+P L_{n, k}^{\min } \\
Q_{n, k}^{f l e x}= & \left(Q G_{n, k}^{\max }-Q G_{n, k}^{\min }\right) \cdot \varphi_{n, k}^{Q G}+Q G_{n, k}^{\min } \\
& -\left(Q L_{n, k}^{\max }-Q L_{n, k}^{\min }\right) \cdot \varphi_{n, k}^{Q L}+Q L_{n, k}^{\min }
\end{aligned}
$$

where $P G_{n, k}^{\max }, P G_{n, k}{ }^{\min }, Q G_{n, k}{ }^{\max }, Q G_{n, k}^{\min }$ are the active and reactive limits of flexibility generation resources. Similarly, $P L_{n, k}{ }^{\max }, P L_{n, k}{ }^{\min }, Q L_{n, k}{ }^{\max }, Q L_{n, k}{ }^{\min }$ are the active and reactive limits of the demand resources. The independent random variables for generation and consumption $\left(\varphi_{n, k}{ }^{P G}, \varphi_{n, k}{ }^{Q G}\right.$, $\left.\varphi_{n, k}{ }^{P L}, \varphi_{n, k^{Q L}}\right)$ are obtained from a uniform distribution between 0 and 1 .

In the second method, the sort of generation and demand flexible resources in the MV nodes ( $n$ ) was no longer independent. In order to obtain coherent active and reactive power injections, a negative correlation between generation and load resources located in the same bus was considered. Equation (3)-(4) presents the calculation of active and reactive power injection in each power flow simulation $(k)$.

$$
\begin{aligned}
P_{n, k}^{f l e x}=\left(P G_{n, k}^{\max }\right. & \left.-P G_{n, k}^{\min }\right) \cdot\left(1-\varphi_{n, k}^{P}\right)+P G_{n, k}^{\min } \\
& -\left(P L_{n, k}^{\max }-P L_{n, k}^{\min }\right) \cdot \varphi_{n, k}^{P}+P L_{n, k}^{\min }
\end{aligned}
$$




$$
\begin{aligned}
Q_{n, k}^{f l e x}=\left(Q G_{n, k}^{\max }\right. & \left.-Q G_{n, k}^{\min }\right) \cdot\left(1-\varphi_{n, k}^{Q}\right)+Q G_{n, k}^{\min } \\
& -\left(Q L_{n, k}^{\max }-Q L_{n, k}^{\min }\right) \cdot \varphi_{n, k}^{Q}+Q L_{n, k}^{\min }
\end{aligned}
$$

As shown in the equations above, the term $\varphi_{n, k}{ }^{P G}, \varphi_{n, k}{ }^{Q G}$, $\varphi_{n, k}^{P L}$ and $\varphi_{n, k}{ }^{Q L}$ were replaced by $\varphi_{n, k}{ }^{P}, \varphi_{n, k}{ }^{Q}$, which means that the same random variable is used to select active and reactive generation.

Active power injections $\left(P_{n, k}^{\text {flex }}\right)$ in PQ and PV buses as well as reactive power injections $Q_{n, k}$ flex in PQ nodes are used to run power flow scenarios.

\section{B. Verification of Network Constraints}

After running a power flow scenario - combining inputs of different random nodal power injections with different tap steps - it is necessary to verify its feasibility.

Consequently, for a given flexibility scenario, an algorithm that checks technical constraints is used to ensure that no line congestions nor voltage violation can occur. Equation (5) and (6) present the verification conditions for line congestion and the voltage violation. If any violation is detected the scenario is ignored and it is not integrated into the PQ region that is communicated to the TSO.

$$
\begin{gathered}
S_{i-j} \in\left[S_{i-j}{ }^{\min }, S_{i-j}^{\text {max }}\right] \\
V_{n} \in\left[V_{n}^{\text {min }}, V_{n}^{\text {max }}\right]
\end{gathered}
$$

in all branches $i-j(5)$

in all buses $n$ (6)

\section{Flexibility Costs}

When the sample operating point does not violate the system limits, the total flexibility cost for that point is calculated according to equation (7). Furthermore, DSO can also consider a flexibility cost associated with the tap change, which can be fixed or proportional to the modification regarding the initial operating point. However, in this work the DSO costs are not considered.

$$
\begin{aligned}
\text { Flexibility }_{\text {Cost }, k}= & \sum_{i=1}^{n}\left|P_{n, k}^{f l e x, G}-P_{G}^{n}\right| \cdot \text { Cost }_{\text {active_flex }} \\
& +\left|Q_{n, k}^{\text {flex }, G}-Q_{G}^{n}\right| \cdot \text { Cost }_{\text {reactive_flex }} \\
& +\left|P_{n, k}^{f l e x, D}-P_{D}^{n}\right| \cdot \text { Cost }_{\text {active_flex }} \\
& +\left|Q_{n, k}^{f l e x, D}-Q_{D}^{n}\right| \cdot \text { Cost }_{\text {reactive_flex }}
\end{aligned}
$$

where $P_{G}^{n} \quad$ represents the active generation estimated/forecasted in bus $\mathrm{n}$ (MW); $Q_{G}^{n}$ represents the operating point estimated/forecasted for the reactive generation in bus $\mathrm{n}$ (MVAr); $P_{D}^{n}$ represents the active consumption estimated/forecasted in bus $\mathrm{n}(\mathrm{MW}) ; Q_{D}^{n}-$ represents the reactive consumption estimated/forecasted in bus n (MVAr); Cost active_flex $_{\text {, Cost }}$ reactive_flex $_{\text {- represents }}$ the fixed cost for remunerate $1 \mathrm{MW} / \mathrm{MV}$ ar of flexibility $(€ / \mathrm{MW}, € / \mathrm{MVAr})$.

\section{CASE Study}

The section aims at demonstrating the application of the methodology proposed above to a real Portuguese MV distribution network with a total of 210 nodes and 212 branches. It is a typical rural network with a radial structure that includes a $60 / 30 \mathrm{kV}$ primary substation and two parallel connected $30 / 15 \mathrm{kV}$ OLTC transformers (with taps on the secondary side of the transformer). A detailed network description can be found in [13].

As shown in Fig. 4 the network is divided in three zones. Zone $1(30 \mathrm{kV})$ contains only consumption nodes with a total demand of $1.978 \mathrm{MW}$ and $0.198 \mathrm{MVAr}$. The generation units are located in zones 2 and $3(15 \mathrm{kV})$ : two wind generators based on DFIGs (one in each zone) and a CHP unit (zone 2). There are also six microgrids injecting power into the MV grid. Tables I and II summarize the generation and load for the scheduled operating point.

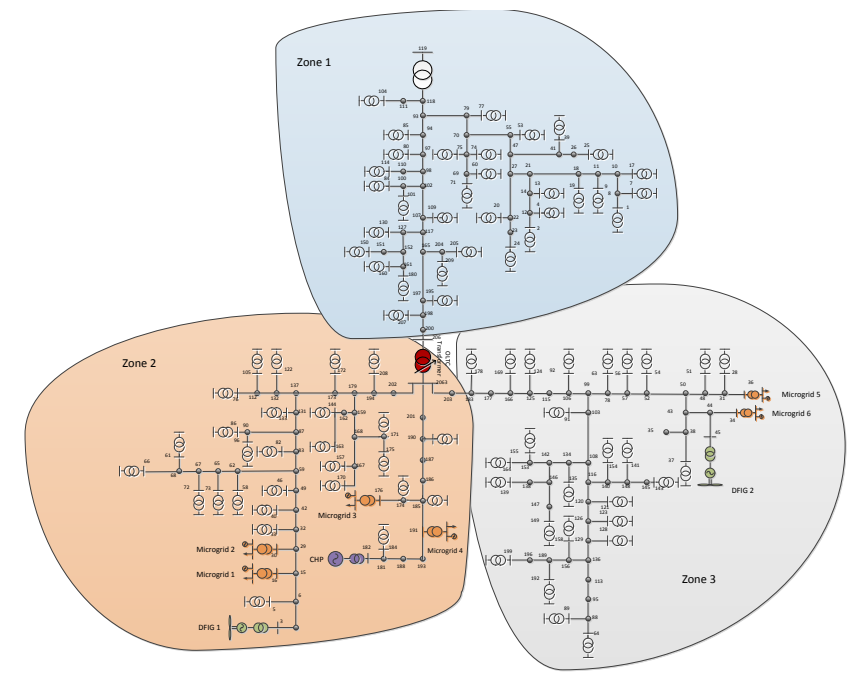

Figure 4. Medium voltage distribution network used test case [13].

TABLE I. Generated Active Power for EACH Zone

\begin{tabular}{|c|c|c|}
\hline Zone & $\boldsymbol{I D}$ & $\boldsymbol{P}_{\boldsymbol{G}}(\boldsymbol{M W})$ \\
\hline \multirow{4}{*}{2} & DFIG1 & 1.000 \\
\cline { 2 - 3 } & CHP & 2.000 \\
\cline { 2 - 3 } & Microgrid1 & 0.082 \\
\cline { 2 - 3 } & Microgrid2 & 0.082 \\
\cline { 2 - 3 } & Microgrid3 & 0.433 \\
\cline { 2 - 3 } & Microgird4 & 0.433 \\
\hline \multirow{3}{*}{3} & DFIG2 & 1.000 \\
\cline { 2 - 3 } & Microgrid5 & 0.017 \\
\cline { 2 - 3 } & Microgrid6 & 0.017 \\
\hline
\end{tabular}

TABLE II. TOTAL LOAD FOR EACH ZONE

\begin{tabular}{|c|c|c|c|}
\hline Zone & $\begin{array}{c}\text { Number of } \\
\text { Loads }\end{array}$ & $\boldsymbol{P}_{\boldsymbol{D}}(\mathbf{M W})$ & $\boldsymbol{Q}_{\boldsymbol{D}}(\boldsymbol{M W})$ \\
\hline 1 & 32 & 1.978 & 0.198 \\
\hline 2 & 33 & 2.223 & 0.222 \\
\hline 3 & 29 & 1.153 & 0.115 \\
\hline Total & $\mathbf{9 4}$ & $\mathbf{5 . 3 5 4}$ & $\mathbf{0 . 5 3 5}$ \\
\hline
\end{tabular}




\section{A) Flexibility estimation without bottleneck}

In this first study, the flexibility estimation algorithm was initially tested in the radial network without a bottleneck in the OLTC transformer and primary substation capacity. However, the branches and voltage limits are considered, but without any effect in constraining the flexibility region.

The active and reactive power flexibility from controllable loads was considered to be within a range of $+/-20 \%$ with respect to the estimated scheduled operating point. A similar range was defined for the active power of generators. However, it has been assumed that the reactive power generation can take any value within the limits of the generation units. Tables III and IV summarize the flexibility of load and generation considered for each zone of the network.

The range of tap positions of an OLTC transformer (flexibility of $+-/ 5 \%$ discretely divided among 10 positions) was also included as a controllable resource of the DSO.

The flexibility cost was assumed to be $50 €$ per MW (or MVAr). For simplicity's sake, the same price was used for both upward and downward directions. Nevertheless, the algorithm is able to deal with a complete range of offers characterized by different prices (e.g., asymmetric prices for upward and downward direction).

TABLE III. ACTIVE AND REACTIVE GENERATION FLEXIBILITY

\begin{tabular}{|c|c|c|c|c|c|}
\hline \multirow{2}{*}{ Zone } & \multicolumn{5}{|c|}{ Active and Reactive Generation Flexibility } \\
\hline & ID & $\begin{array}{l}P G^{\min } \\
(M W)\end{array}$ & $\begin{array}{l}P G^{\max } \\
(M W)\end{array}$ & $\begin{array}{c}Q G^{\min } \\
(\text { Mvar) }\end{array}$ & $\begin{array}{c}Q G^{\max } \\
\text { (Mvar) }\end{array}$ \\
\hline \multirow{6}{*}{2} & DFIG1 & 0.8000 & 1.2000 & -0.1000 & 0.2000 \\
\hline & CHP & 1.6000 & 2.4000 & -0.4000 & 0.8000 \\
\hline & Microgrid1 & 0.0656 & 0.0984 & 0.0000 & 0.0000 \\
\hline & Microgrid2 & 0.0656 & 0.0984 & 0.0000 & 0.0000 \\
\hline & Microgrid3 & 0.3464 & 0.5196 & 0.0000 & 0.0000 \\
\hline & Microgird4 & 0.3464 & 0.5196 & 0.0000 & 0.0000 \\
\hline \multirow{3}{*}{3} & DFIG2 & 0.8000 & 1.2000 & -0.1000 & 0.2000 \\
\hline & Microgrid5 & 0.0138 & 0.0208 & 0.0000 & 0.0000 \\
\hline & Microgrid6 & 0.0138 & 0.0208 & 0.0000 & 0.0000 \\
\hline
\end{tabular}

TABLE IV. ACTIVE AND REACTIVE LOAD FLEXIBILITY

\begin{tabular}{|c|c|c|c|c|}
\hline \multirow{2}{*}{ Zone } & \multicolumn{4}{|c|}{ Active and Reactive Load Flexibility } \\
\hline & $P L^{\min }(M W)$ & $P L^{\max }(M W)$ & $\begin{array}{c}Q L^{\min } \\
\text { (Mvar) }\end{array}$ & $\begin{array}{c}Q L^{\max } \\
(\text { Mvar) }\end{array}$ \\
\hline 1 & 1.5824 & 2.3736 & 0.1584 & 0.2376 \\
\hline 2 & 1.7784 & 2.6676 & 0.1776 & 0.2664 \\
\hline 3 & 4.2832 & 6.4248 & 0.4280 & 0.6420 \\
\hline Total & 4.2832 & 6.4248 & 0.4280 & 0.6420 \\
\hline
\end{tabular}


load and generation for the same initial operating point. Indeed, in the independent selection of flexible resources, "central" values of injected power can be obtained through a combination of extreme flexibilities, which increases the total cost. For example, a small change in the primary substation operating point can be obtained either by activating a small amount of flexibility or by dispatching, at the same network of the node, significant upward generation and downward load (or vice versa). Although the operating point modification is equivalent, the second option is more expensive. However, in the independent selection of flexible resources both situations occur. Therefore, a huge number of samples is required in order to obtain the lowest costs of the system flexibility. Hence, as shown in Fig. 5 after 100,000 iterations no flexibility cost lower than $25 € / \mathrm{h}$ was found.

In contrast, forcing a negative correlation between generation and consumption, with a lower number of samples is possible to cover a wider range of flexibility costs, obtaining also lower values.

\section{B. Flexibility estimation with bottleneck}

This subsection shows the method' results in a scenario with a potential bottleneck, i.e., the branches of the MV distribution network are near their congestion capacity. Therefore, an extreme scenario was considered: the OLTC transformer capacity was reduced to $0.85 \mathrm{MVA}$ and the limit of the primary substation transformer was assumed to be 0.80 MVA.

Fig. 7 illustrates the application of the proposed method to this new scenario, considering the negative correlated selection of the flexible resources. By comparing this figure with Fig. 6, it is possible to see that the DSO network flexibility available at the primary substation decreases dramatically because the capacity of the transformer does not allow more flexibility. In fact, although similar costs were obtained, a more narrowed feasible flexibility range can be offered to the TSO.

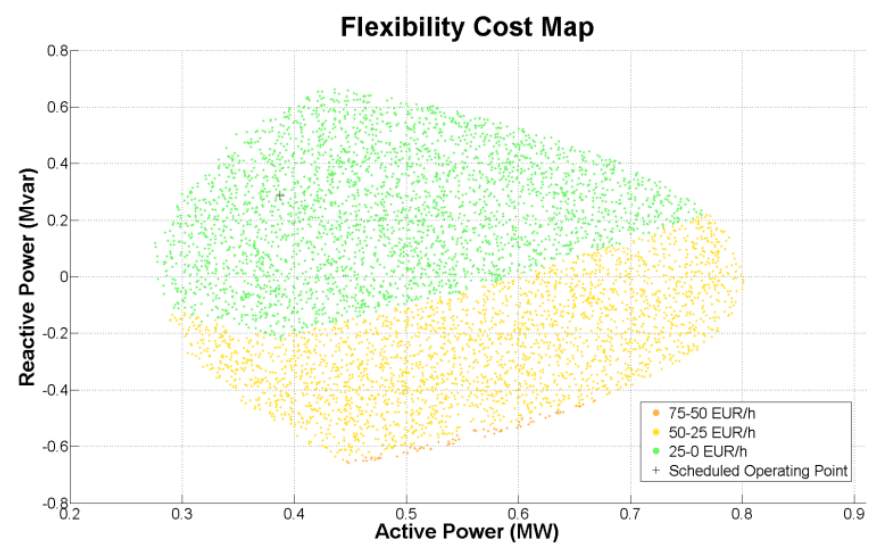

Figure 7. Flexibility Cost Map considering branch limits.

\section{CONCLUSIONS}

The results presented in this paper represent a first step towards a more proactive coordination between TSO and DSO within the smart grid concept. The proposed tool provides the TSO with information about the technically available flexibly in the distribution system, which, as shown in the results section, can be constrained by the physical limits of the network. This type of information is particularly useful in operating scenarios characterized by reverse power flows and uncertain renewable power injections.

The random sampling using the Monte Carlo simulation is not the better solution since it is difficult to capture the extreme (but feasible) flexibility points of the primary substation even increasing the number of samples. Therefore, an idea for future research consists in formulating an optimization model to solve this problem, as well as to reduce the computational effort need to identify the flexibility area. Moreover, other type of flexibilities should also be included in the methodology, such as Flexible AC Transmission Systems (FACTS) and distributed storage, as well as asymmetric flexibility costs.

\section{REFERENCES}

[1] H. Farhangi, "The path of the smart grid," IEEE Power and Energy Magazine, vol. 8(1), pp. 18-28, Jan.-Feb. 2010.

[2] C.S. Gouveia, D. Rua, F.J. Soares, C. Moreira, P.G. Matos, J.A. Peças Lopes, "Development and implementation of Portuguese smart distribution system," Electric Power Systems Research, vol. 120, pp.150-162, Mar. 2015.

[3] D. Pudjianto, C. Ramsay and G. Strbac, "Virtual power plant and system integration of distributed energy resources," IET Renewable Power Generation, vol. 1(1), pp. 10-16, Mar. 2007.

[4] R. Schwerdfeger and D. Westermann, "Storage management algorithm for schedule based DSO system operation with respect to TSO's system responsibility," in Proc. of the 18th Power Systems Computation Conference, Wroclaw, Poland, Aug. 18-22, 2014.

[5] ENTSO-E, Network Code on Operational Planning and Scheduling, Final Version, Sept. 2013.

[6] A. Ulian, M. Sebastian, G. Bartolucci, C. Gutschi, "Business use cases definition and requirements," Deliverable D2.1, EU Project evolvDSO, Jul. 2014.

[7] E. Rivero, S. Daan, A. Ramos, M. Maenhoudt, A. Ulian, "Preliminary assessment of the future role of DSOs, future market architectures and regulatory frameworks for network integration of DRES," Deliverable D1.3, EU Project evolvDSO, Jul. 2014.

[8] P. Oosterkamp, P. Koutstaal, A. van der Welle, J. de Joode, et al., "The role of DSOs in a Smart Grid environment," Final Report for the European Commission and DG ENER, April 2014.

[9] R.J. Bessa, A. Trindade, V. Miranda, "Spatial-temporal solar power forecasting for Smart Grids," IEEE Transactions on Industrial Informatics, vol. 11(1), pp. 232-241, Feb. 2015.

[10] Council of European Energy Regulators, "Regulatory and market aspects of demand-side flexibility," CEER Public Consultation Document, Nov. 2013.

[11] G. Bartolucci "System Use Cases definition and functional and nonfunctional requirements for tools and methods, and definition of KPIs," Deliverable D2.2, EU Project evolvDSO, Oct. 2014.

[12] IntelliGrid methodology for developing requirements for energy systems, IEC/PAS 62559 ed1.0, Jan. 2008.

[13] A. Madureira, "Coordinated and optimized voltage management of distribution networks with multi-microgrids", Ph.D. Thesis, Faculty of Engineering of the University of Porto (FEUP), Jul. 2010. 